\section{Aktuelle Urteile}

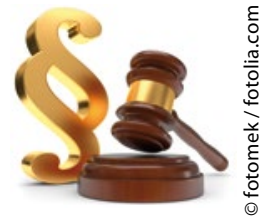

\section{Verfassungsrichter finden kein schlechtes Haar am G-BA}

Das Bundesverfassungsgericht in Karlsruhe hat einen Angriff gegen den Gemeinsamen Bundesausschuss der Ärzte und Krankenkassen (G-BA) abgewiesen. Das mächtige Gremium entscheidet über den Leistungskatalog der gesetzlichen Krankenkassen, ist aber in der Öffentlichkeit weitgehend unbekannt. In einer aktuellen Beschwerde war deshalb auch wieder die unzureichende demokratische Legitimation des Ausschusses beanstandet worden.Den Richtern war das zu allgemein. Eine Verfassungsbeschwerde müsse unmittelbar auf einzelne Entscheidungen abzielen. Maßgeblich sei dann, ob diejenigen, die von der Entscheidung am stärksten betroffen sind, ausreichend daran mitwirken konnten. Zudem komme es darauf an, wie eng die gesetzlichen Vorgaben für die Entscheidung sind. Beides aber könne von Regelung zu Regelung unterschiedlich sein.

- mwo/chy

- BVerfG, Az.: 1 BVR 2056/12

\section{Kasse muss bei Schmerzen Brustverkleinerung bezahlen}

Verursachen besonders große Brüste bei einer Frau anhaltend starke Rücken- und Nackenschmerzen, kann sie von ihrer Krankenkasse die Kostenübernahme für eine Brustverkleinerungs-Operation verlangen. Das gilt jedenfalls dann, wenn sich auch bei starker Gewichtsabnahme die Brust nicht mit verkleinert, entschied das Sozialgericht Aachen. Geklagt hatte eine stark übergewichtige, 1,62 Meter große Frau, die über einen Zeitraum von acht Monaten $30 \mathrm{~kg}$ abgenommen hatte. Das Gewicht ihrer Brüste, Körbchengröße F, verringerte sich dabei aber nur unwesentlich. Wegen starker Schmerzen befürworteten Frauenarzt und Orthopäde eine operative Brustreduktion. Der Gutachter des Medizinischen Dienstes der Krankenkassen (MDK) entschied nach Aktenlage und lehnte eine Kostenübernahme ab. Die Frau müsse erst einmal ein halbes Jahr weiter abnehmen und einen Body-Mass-Index von unter 30 erreichen. Die Klägerin bezahlte die Operation selbst - und bekam vom Gericht nun die Kostenerstattung in Höhe von 6.732 Euro zugesprochen.

- $\mathrm{fl} / \mathrm{mwo}$ meinen Mann in Notfällen informieren." Bis heute arbeitet sie in der Hadmerslebener Praxis mit, die nun allerdings ein MVZ mit insgesamt 25 Beschäftigten und fünf Außenstellen ist.

Derzeit investieren die drei MVZ-Gesellschafter - neben Uwe Milbradt zwei weitere Ärzte - in einen Neubau im benachbarten Wanzleben. Hier wollen sie ein Domizil für ihr ambulantes Geriatrisches Rehabilitationszentrum schaffen, das sie im April dieses Jahres eröffnet haben. Außerdem ist noch Platz für physio- und ergotherapeutische Praxen.

Schon heute lässt sich absehen: Das Konzept geht auf. „Gemeinsam geht eben vieles besser", meint Milbradt, dem es auch nie gleichgültig war, wenn Poliklinik-Ärzte ihm zu DDR-Zeiten ankrei- deten, weitaus mehr Patienten zu haben. „Dabei hatten sie im Gegensatz zu mir ein festes Gehalt und kein wirtschaftliches Risiko."

Wohl auch, weil ihm das Konkurrenzgehabe immer gewaltig auf die Nerven ging, entschied sich der Landarzt gleich nach der Wende für eine Gemeinschaftspraxis. „Ich hatte Anfang 1984 nach vielen Irrungen und Wirrungen die Praxis meines verstorbenen Vaters übernehmen dürfen und war seither in freier Niederlassung." Im Gegensatz zu den meisten Kollegen, die in Krankenhäusern, Polikliniken oder Ambulatorien angestellt waren, konnte Milbradt so bereits einige Erfahrungen weitergeben - und vor allem Mut machen.

Petra Zieler

\title{
Oskar Medizinpreis 2015 verliehen
}

Den Oskar Medizinpreis 2015, den die Berliner Stiftung Oskar-Helene-Heim vergibt, teilen sich Prof. Julia-Stefanie Frick, Tübingen, und PD Dr. Anja Sandek, Göttingen. Sie werden für wegweisende Forschungen zu den Auswirkungen des Mikrobioms im Gastrointestinaltrakt geehrt. Der Preis ist mit insgesamt 50.000 Euro dotiert.

In den letzten Jahren hat es eine Vielzahl von Hinweisen auf metabolische, immunologische, infektiologische, neurologische und epidemiologische Einflussfaktoren des Mikrobioms auf die Pathophysiologie verschiedener Erkrankungen ergeben. Frick beforscht die Interaktion von kommensalen Bakterien und dem Wirt und ihren Einfluss auf lokale entzündliche und infektiöse Wirtsantworten wie auch systemische Immunantworten. Sandeks Thema ist die Bedeutung von Gastrointestinaltrakt und Mikrobiom für die Entstehung von systemischen Entzündungen bei Patienten mit chronischer Herzinsuffizienz.

Mitglieder der Jury waren Prof. Hans-Dieter Allescher, Garmisch-Partenkirchen, Prof. Peter Bauerfeind, Zürich, Prof. Thomas Frieling, Krefeld, Prof. Christoph Högenauer, Graz, und Prof. Stefan Müller-Lissner, Berlin.

- Red.

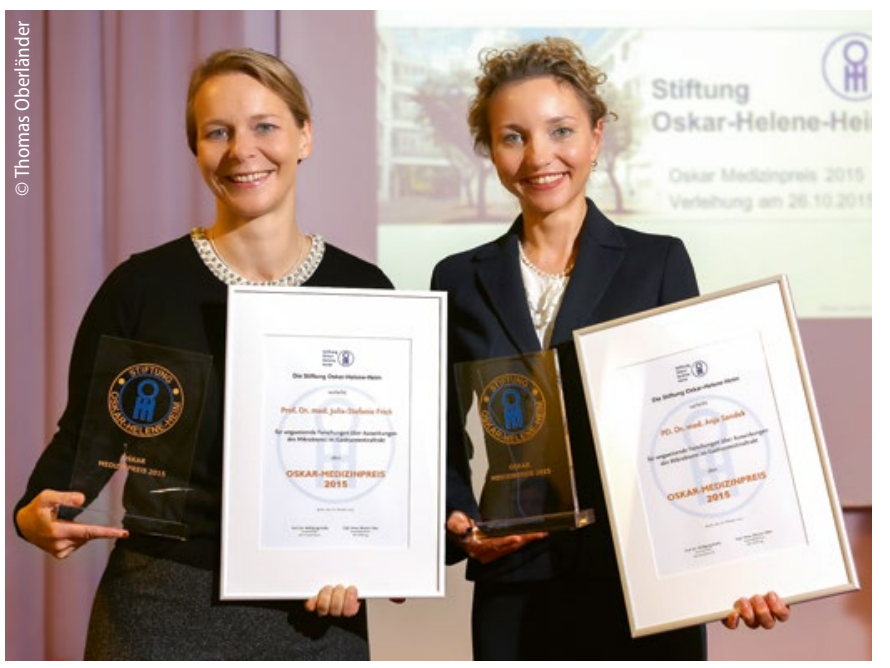

In diesem Jahr teilen sich Prof. Julia-Stefanie Frick und PD Dr. Anja Sandek den Oskar Medizinpreis 2015. 